\title{
On distortion of the background radiation spectrum by wormholes: kinematic Sunyaev-Zel'dovich effect
}

\author{
A.A. Kirillov ${ }^{1}$ and E.P. Savelova ${ }^{1}$
}

Received —

\footnotetext{
${ }^{1}$ Bauman Moscow State Technical University, Moscow, 105005, Russia
} 


\begin{abstract}
The problem of scattering of the background radiation on relic cosmological wormholes is considered. It is shown that static wormholes do not perturb the spectrum at all. The presence of peculiar velocities of wormholes results in a distortion of the CMB spectrum which is analogous to the kinematic SunyaevZel'dovich effect. In the first order in $v / c$ the distortion of CMB cannot be separated from from the Compton scattering on electrons. In next orders the scattering on wormholes exhibits some difference from the Compton scattering. High-energy cosmic-ray particles spectrum does not change the form by KSZ, but undergoes a common Doppler shift. Such features may give a new tool to detect the presence of relic wormholes in our Universe.
\end{abstract}

Subject headings: CMB, cosmic rays, kinematic Sunyaev-Zel'dovich effect, cosmological wormholes 


\section{Introduction}

As it was recently shown some basic difficulties of cold dark matter models $(\Lambda \mathrm{CDM})$ can be cured by the presence of relic cosmological wormholes (Kirillov \& Savelova 2011, 2016, 2017). To avoid misunderstanding we point out that relic wormholes are not going to replace completely the dark matter paradigm, since there exist phenomena related to dark matter which wormholes unable to explain. The existence of relic wormholes however is not in a conflict with the simultaneous existence of dark matter particles, the so-called WIMPs (weakly interacting massive particles). Save the dark matter phenomena observed in astrophysics (dark matter halos in galaxies, CMB spectrum, observed structures, etc.), the presence of WIMPs is well motivated by numerous problems of the Standard Model in particle physics, e.g., see the list in (Feng 2010). In particular, the observed high-energy cosmic-ray electrons and positrons (Ambrosi et al. 2017) may enable the observation of phenomena such as dark-matter particle annihilation or decay (Grib \& Pavlov 2009). Relic wormholes do not produce such an effect, though spherically symmetric wormholes collapse and form black holes and may produce all astrophysical phenomena related to them.

We however point out that WIMPs may have the direct relation to virtual wormholes. Such wormholes have virtual character and describe quantum topology fluctuations (Savelova 2015, 2016). It was shown recently in (Kirillov \& Savelova 2015) that for all types of relativistic fields, the scattering on virtual wormholes leads to the appearance of additional very heavy particles, which play the role of auxiliary fields in the invariant scheme of Pauli-Villars regularization. In the simplest picture the mas spectrum of such additional particles starts from the Planck value $M_{p l}$ and is completely determined by parameters of the vacuum distribution of virtual wormholes. It is important that such additional particles are generated for all sorts of particles in the Standard Model and have a discrete spectrum of the more increasing masses. For example, standard massless photons are accompanied 
with massive photons with masses $M_{i}=a_{i} M_{p l}$, where coefficients $a_{i}\left(a_{1}<a_{2}<\ldots\right)$ are expressed via the distribution of virtual wormholes (Kirillov \& Savelova 2015). On the very early stage of the development of the Universe such particles were in an abundance in the primordial hot plasma. During the expansion the Universe cools and most of such particles decay. At least all such particles decay if they are involved in the strong or electromagnetic interactions. However weakly interacting particles may survive till the present days and they indeed may play the role of dark matter (e.g., extremely massive gravitons, neutrinos, etc.).The decay of such superheavy particles into unstable particles with large mass is described by (Grib \& Pavlov 2009), while their subsequent decay into quarks and leptons leads to events in cosmic rays. In particular, the detected break in the teraelectronvolt cosmic-ray spectrum of electrons and positrons (Ambrosi et al. 2017) can be interpreted as the trace of the decay of two sorts of such particles with different masses $M_{1} \ll M_{2}$. The values $M_{i}$ determine the absolute boundaries of the respective spectra, while the factor $\exp \left(-\frac{\Delta M}{T_{c}}\right)$ (where $T_{c}$ is the temperature at which the primordial content of such particles had been tempered) determines the ratio of the respective number of events. One may expect that analogous break should be observed and for higher energies as well.

In astrophysical picture relic wormholes produce also a number of effects analogous to effects from dark matter particles and, therefore, the number density of such particles in galactic halos may essentially change, when the presence of relic wormholes is taken into account. Indeed, as it was demonstrated by (Kirillov \& Savelova 2011) at very large scales wormholes contribute to the matter density perturbations exactly like standard cold dark matter particles and do not destroy all predictions of $\Lambda$ CDM models. However at smaller sub-galactic scales wormholes strongly interact with all existing particles. They scatter photons, baryons, and dark matter particles and, therefore, they do smooth away cusps predicted by numerical simulations at galactic centers (Navarro, Frenk, and White 1996). Recall that cold heavy particles unavoidably form cusps $\rho_{D M} \sim 1 / r$, while observations 
(Gentile et.al. 2004; Borriello et.al. 2003; Weldrake et. al. 2003) show not such a feature. This may be considered as an essential indirect argument in favor of the existence of relic wormholes, since all other known mechanisms of removing cusps are not efficient.

We point also out that strong theoretical arguments for the existence of relic wormholes come from lattice quantum gravity (Ambjorn et al. 2005). Indeed, it is assumed that at Planckian scales the topological structure of our Universe should have fractal properties. During the inflationary stage the topological structure of space should temper and may survive till the present days in the form of relic cosmological wormholes. The problem of the formation of relic wormholes is not described rigorously yet and we do not discuss it here. Nevertheless, some hints on such a picture can be found from the distribution of galaxies. On scales below $100 \mathrm{Mp}$ the distribution of galaxies definitely shows fractal features (Labini et.al. 1998), see also the more resent results in (Conde-Saavedraa et al. 2014). Such a structure may serve as a direct trace of the actual topological structure of space. Indeed, if we assume the homogeneous distribution of galaxies then the number counts $N(R) \sim R^{D}$ (where $N(R)$ is the number of galaxies within the radius $R$ and $D$ is the dimension) reflects the behavior of the physical volume of space. It crosses over to the homogeneity only on larger scales (Planck Collaboration 2015a, b) which however cannot rule out the possibility of the existence of relic wormholes. Indeed, as it was shown by (Battistelli et.al. 2016) in the absence of peculiar velocities wormholes do not perturb spectrum of the cosmic background radiation and, therefore, they cannot be distinguished on the sky. The detection of relic wormholes requires studying more subtle effects. Of the primary interest are those effects which can be disentangled from effects produced by black holes and other forms of matter.

In the present paper we consider the scattering of background radiation on wormholes and show that they can be in principle observed by means of the effect analogous to the 
kinematic Sunyaev-Zel'dovich effect (KSZ) (Sunyaev \& Zeldovich 1980a, , ba). KSZ signal is based on the inverse Compton scattering of relic photons on a moving electron gas. It represents one of the main tools in studying peculiar motions of clusters and groups of galaxies, e.g., see (Kashlinsky et al. 2011; Savers et. al. 2013; Hand et.al. 2012), and see also more applications in a recent review (Battistelli et.al. 2016). It is actually produced by any kind of matter which scatters CMB (not only by a hot electron gas). As it was shown in (Battistelli et.al. 2016), in the first order in $V / c$ the contribution of wormholes into KSZ cannot be separated from that of the electron gas in clusters and groups. Therefore, there are two possibilities. First one is to look for such an effect in those spots on the sky where the baryonic matter is absent, e.g., in voids where the leading contribution will come from wormholes alone. In this case however we need also some additional independent effects to be sure that the signal comes from a void and not from the last scattering sphere. The second possibility is to study next order corrections and peculiar features of the scattering of background radiation on wormholes. In the case of CMB it turns out that already in the second order KSZ effect on wormholes differs from that on other sorts of matter. In the case of high-energy cosmic rays KSZ produces simply a shift of spectrum without the change of it's form.

The most simple wormhole is described by a spherically symmetric configuration. Spherical wormholes can be made stable only by the presence of exotic matter (Hochberg \& Visser 1998). While the natural sources of the exotic matter are not found, we should state that all relic spherical wormholes collapse very rapidly and hardly survive till the present days. If this occur at relatively late time compared to the time of photon decoupling, then emissions from collapsed structures may contribute to the cosmic-ray background which is different from CMB (e.g., infrared, X-ray, etc.). Remnants of such spherical wormholes can not be distinguished from ordinary primordial black holes and we do not discuss them here. However, as it was shown recently by (Kirillov \& Savelova 
2016) stable relic wormholes may exist without exotic matter, if they have a less symmetric structure. The rate of evolution of such wormholes is comparable with the rate of cosmological expansion and, therefore, such wormholes may survive till the present days. The less symmetric wormholes have throat sections in the form of a torus or even more complicates surfaces (Kirillov \& Savelova 2016). We use a torus-like wormhole in considering some peculiar features of the scattering on a single wormhole is the section 4 . Torus-like wormholes have random orientations in space. Upon averaging over orientations the torus-like wormhole acquires features of a spherically symmetric configuration. This allows us to use spherical wormholes in considering estimates for KSZ and the second order corrections to KSZ.

\section{Cross-sections and KSZ effect}

The scattering of electromagnetic waves on a spherical wormhole has been considered first by (Clement 1984; Perez Bergliaffa \& Hibberd 2000). There are two important features of such a scattering which are the generation of a specific interference picture upon scattering on a single wormhole (Kirillov \& Savelova 2012) and the generation of a diffuse halo around any discrete source (Kirillov et.al. 2009). If a wormhole is not very big, the interference picture gives too weak signal and, therefore, it can be used only in the future observations. The generation of the diffuse halo around discrete sources may have various interpretations and this results in a difficulty to disentangle effects of wormholes and, for example, effects of the scattering on dust.

The simplest model of a spherical wormhole is given by a couple of conjugated spherical mirrors, when a relict photon falls on one mirror it is emitted, upon the scattering, from the second (conjugated) mirror. Such mirrors represent two different entrances into the wormhole throat and they can be separated by an arbitrary big distance in the outer space. 
The cross-section of such a process has been described by (Kirillov \& Savelova 2012) and can be summarized as follows. Let an incident plane wave (a set of photons) falls on one throat. Then the scattered signal has two components. The first component represents the standard diffraction (which corresponds to the absorption of CMB photons on the throat) and forms a very narrow beam along the direction of the propagation. This is described by the cross-section

$$
\frac{d \sigma_{a b s o r}}{d \Omega}=\sigma_{0} \frac{(k a)^{2}}{4 \pi}\left|\frac{2 J_{1}(k a \sin \chi)}{k a \sin \chi}\right|^{2},
$$

where $\sigma_{0}=\pi a^{2}, a$ is the radius of the throat, $k$ is the wave vector, and $\chi$ is the angle from the direction of propagation of the incident photons, and $J_{1}$ is the Bessel function. Together with this part the second throat emits an omnidirectional isotropic flux with the cross-section

$$
\frac{d \sigma_{e m i t}}{d \Omega}=\sigma_{0} \frac{1}{4 \pi}
$$

The both total cross-sections coincide

$$
\int \frac{d \sigma_{\text {absor }}}{d \Omega} d \Omega=\int \frac{d \sigma_{\text {emit }}}{d \Omega} d \Omega=\sigma_{0}
$$

which expresses the conservation law for the number of absorbed and emitted photons. In the absence of peculiar motions (a static gas of wormholes) every wormhole throat end absorbs photons as the absolutely black body, while the second end re-radiates them in an isotropic manner (2) with the same black body spectrum. It is clear that there will not appear any distortion of the CMB spectrum at all. In the presence of peculiar motions the motion of one end of the wormhole throat with respect to CMB causes the angle dependence of the incident radiation with the temperature

$$
\begin{gathered}
T_{1}=\frac{T_{C M B}}{\sqrt{1-\beta_{1}^{2}}\left(1+\beta_{1} \cos \theta_{1}\right)} \simeq \\
T_{C M B}\left(1-\beta_{1} \cos \theta_{1}+\frac{1}{2}\left(1+2 \cos ^{2} \theta_{1}\right) \beta_{1}^{2}+\ldots\right)
\end{gathered}
$$


where $\beta_{1}=V_{1} / c$ is the velocity of the throat end and $\beta_{1} \cos \theta_{1}=\left(\vec{\beta}_{1} \vec{n}\right), \vec{n}$ is the direction for incident photons. Therefore, the absorbed radiation has the spectrum

$$
\begin{aligned}
\rho\left(T_{1}\right) & =\rho\left(T_{C M B}\right)+\frac{d \rho\left(T_{C M B}\right)}{d T} \Delta T_{1}+ \\
& +\frac{1}{2} \frac{d^{2} \rho\left(T_{C M B}\right)}{d T^{2}} \Delta T_{1}^{2}+\ldots,
\end{aligned}
$$

where $\rho(T)$ is the Planckian spectrum and $\Delta T_{1}=T_{1}-T_{C M B}$. As it was discussed previously by (Battistelli et.al. 2016) in the first order in $\beta_{1}$ the above anisotropy does not contribute to the re-radiation of relic photons from the second end according to (2). Indeed, integration over the incident angle $\theta_{1}$ gives $\langle\Delta T\rangle=-\frac{1}{4 \pi} \int \beta_{1} \cos \theta_{1} d \Omega=0$. In other words, in the firs order in $\beta_{1}$ the peculiar motions of the absorbing ends of wormholes can be ignored. In this case the KSZ effects caused by wormholes and by the standard baryonic matter mix and cannot be disentangled. The difference however appears in the second order in $\beta_{1}$. Indeed, considering the second order we find

$$
\begin{aligned}
(\Delta \rho)_{2} & =T_{C M B} \frac{d \rho\left(T_{C M B}\right)}{d T} \frac{\left(1+2\left\langle\cos ^{2} \theta_{1}\right\rangle\right)}{2} \beta_{1}^{2}+ \\
& +\frac{T_{C M B}^{2}}{2} \frac{d^{2} \rho\left(T_{C M B}\right)}{d T^{2}}\left\langle\cos ^{2} \theta_{1}\right\rangle \beta_{1}^{2}
\end{aligned}
$$

where $(\Delta \rho)_{2}=\left\langle\rho\left(T_{1}\right)-\rho\left(T_{C M B}\right)\right\rangle_{2}$ which gives

$$
(\Delta \rho)_{2}=\frac{\beta_{1}^{2}}{6 T_{C M B}^{3}} \frac{d}{d T}\left(\frac{d \rho\left(T_{C M B}\right)}{d T} T_{C M B}^{5}\right),
$$

where we used $\left\langle\cos ^{2} \theta_{1}\right\rangle=\frac{1}{3}$. This means that together with the standard Planckian spectrum $I\left(T_{C M B}\right)=c \rho(x)=I_{0} \frac{x^{3}}{e^{x}-1}$, where $I_{0}=\frac{2 h}{c^{2}}\left(\frac{k_{B} T_{C M B}}{h}\right)^{3}$ and $x=h \nu / k_{B} T_{C M B}$, every wormhole emits the additional isotropic flux of photons with the spectrum

$$
\left(\frac{\Delta I}{I_{0}}\right)_{2}=\beta_{1}^{2}=f(x) \beta_{1}^{2},
$$

where $f(x)=\frac{1}{6} \frac{x^{4} e^{x}\left(3 e^{x}-3+x\left(e^{x}+1\right)\right)}{\left(e^{x}-1\right)^{3}}$. We point out that in this case the distortion of the spectrum does not reduce to a frequency-invariant shift of the temperature. For the sake of 


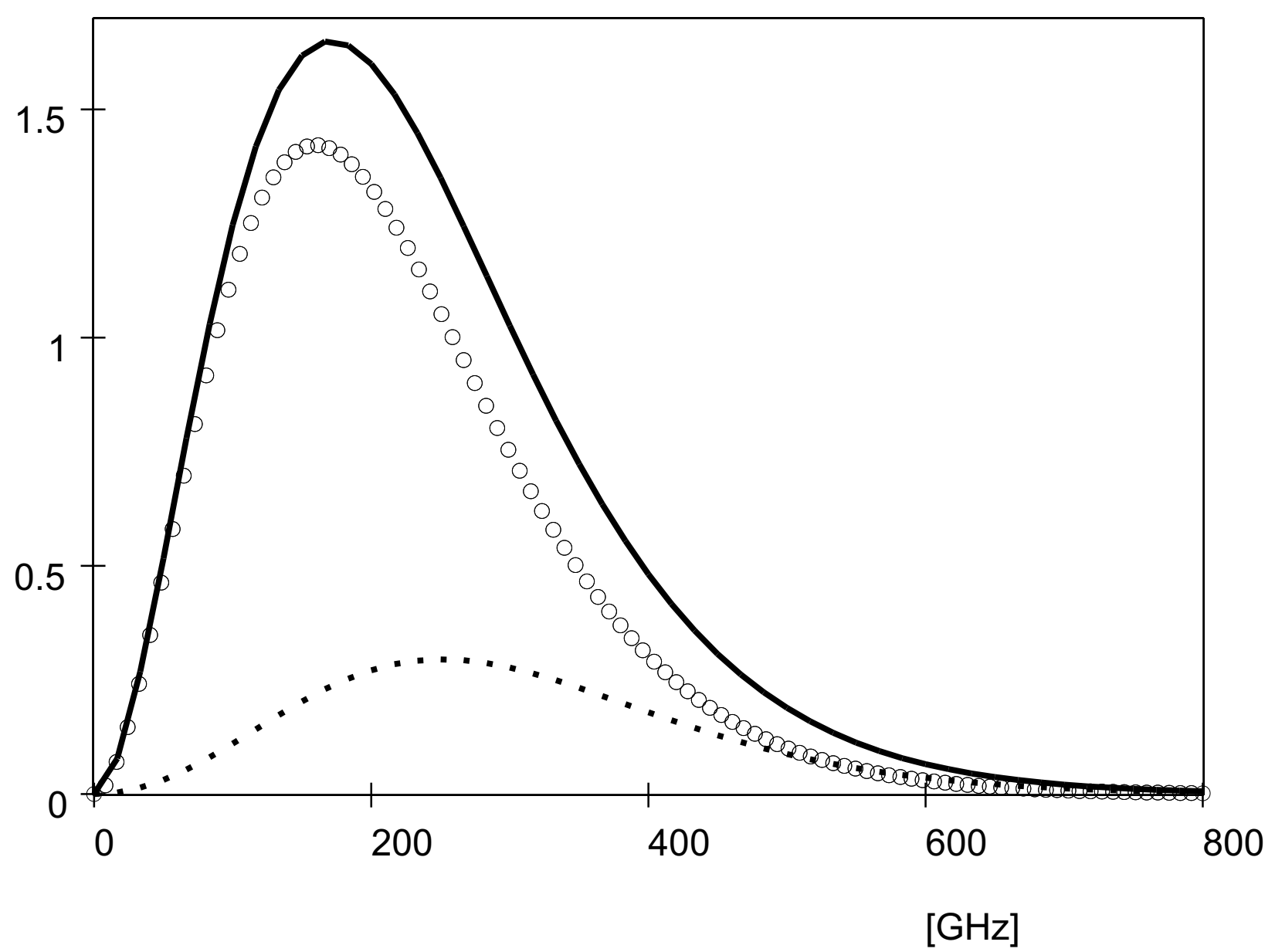

Fig. 1. - Spectra emitted by a single wormhole. In circles is the standard CMB, dotted line is the additional flux (3) for $\beta_{1}^{2}=0.05$, and the solid line is the sum. 
comparison we plot the function $f(x)$ (dotted line for $\beta_{1}^{2}=0.05$ ), the standard Planckian spectrum (in circles) and the sum (solid line) on Fig.1. The estimate of the relative integrated amplitudes of the radiation emitted by a single wormhole is given by

$$
\frac{(\Delta I)_{2}}{I_{C M B}}=5.33 \times \beta_{1}^{2} .
$$

When considering a cloud of wormhole throats, in addition to the standard CMB every throat radiates photons with the flux $(\Delta I)_{2}$. In the presence of peculiar velocities the CMB part undergoes the Doppler shift (which is the complete analog of the KSZ effect)

$$
\frac{\Delta T_{K S Z}}{T_{C M B}}=\beta_{p} \tau_{w}
$$

Here $\beta_{p}$ is the projection of the peculiar velocity of the cloud along the line of sight and the optical depth $\tau_{w}$ defined as

$$
\tau_{w}=\int \pi a^{2} n(r, a) d a d \ell
$$

where the integration is taken along the line of sight and $n(r, a)$ is the number density of wormholes measured from the center of the cloud and depending on the throat radius $a$. The optical depth $\tau_{w}$ is interpreted as follows. Let $L$ be the characteristic size of the cloud of wormholes. Then on the sky it will cover the surface $S \sim L^{2}$, while the portion of this surface covered by wormhole throats is given by

$$
\tau_{w}=\frac{N \pi \overline{a^{2}}}{L^{2}}=\pi \overline{a^{2}} \bar{n} L
$$

where $N$ is the number of wormhole throats in the cloud and $\bar{n}$ is the mean density. In a sufficiently dense cloud $\tau_{w} \sim 1$ this effect produces simply a hot or a cold (depending on the sign of $\beta_{p}$ ) spot on the CMB maps. It is important that KSZ corresponds to a frequency-invariant temperature shift which leaves the primary CMB spectrum unchanged.

The second order effect discussed earlier does not depend on velocities of throats in the cloud. It however depends on velocities of conjugated entrances into throats and is given by

$$
\frac{(\Delta I)_{2 K S Z}}{I_{C M B}}=5.33 \times\left\langle\beta_{1}^{2}\right\rangle \tau_{w}
$$


where $\left\langle\beta_{1}^{2}\right\rangle \tau_{w}=\int \beta_{1}^{2} \pi a^{2} n\left(r, a, \beta_{1}^{2}\right) d a d \ell d \beta_{1}$. In general, such an effect is very small, since the typical values does not exceed $\left\langle\beta_{1}^{2}\right\rangle \sim 10^{-4}$. It is however measurable for sufficiently dense clouds $\tau_{w} \sim 1$ and which is important it cannot be reduced to a shift of CMB temperature and, therefore, it does slightly change the primary CMB spectrum according to (3). This gives a new tool which allows to distinguish the contribution of wormholes into KSZ effect from that of the rest matter.

\section{Cosmic-ray spectrum and KSZ effect}

Measurements of the High-energy cosmic-ray spectrum of electrons and positrons is described by a smoothly broken power- law model, e.g., see (Ambrosi et al. 2017)

$$
\Phi(E)=\Phi_{0}\left(\frac{E_{0}}{E}\right)^{\gamma_{1}}\left[1+\left(\frac{E}{b E_{0}}\right)^{\frac{\gamma_{2}-\gamma_{1}}{\Delta}}\right]^{-\Delta}
$$

where $\Delta=0.1, \Phi_{0}=A / E_{0}, E_{0}=100 \mathrm{GeV}, A=(1.64 \pm 0.01) \times 10^{-2} m^{-2} s^{-1} s r^{-1}$, $b=9.14 \pm 0.98, \gamma_{1}=3.09 \pm 0.01$, and $\gamma_{2}=3.92 \pm 0.20$. It shows that at energies $E \simeq E_{b}=b E_{0}$ the spectral index changes from $\gamma_{1} \approx 3.1$ to $\gamma_{2} \approx 3.9$ (Ambrosi et al. 2017). The cross-section described in the previous section works in the case of rays as well. First we point out that the presence of relic wormholes leads to the formation of a diffuse halo (of a low intensity) around any discrete source. When wormholes do not move in space, then they do not change the spectrum at all (Kirillov et al. 2008). Consider now an incident on a wormhole particle. In the rest frame of the wormhole the energy of the incident particle changes according to the standard Lorentz transformation. For High-energy particles it gives

$$
\begin{gathered}
E^{\prime}=\frac{E+(V p)}{\sqrt{1-\beta^{2}}} \simeq E\left(1+\beta \frac{c p}{E} \cos \theta\right) \simeq \\
\simeq E(1+\beta \cos \theta) .
\end{gathered}
$$


Here $\cos \theta$ is the angle between the direction of the incident particle and the velocity of a wormhole entrance. Thus the change of the energy of the particle is given by

$$
\begin{gathered}
\frac{\Delta E^{\prime}}{E}=\frac{1}{\sqrt{1-\beta^{2}}}\left(1+\beta \sqrt{1-\frac{m^{2} c^{4}}{E^{2}}} \cos \theta\right)-1 \\
\frac{\Delta E^{\prime}}{E} \simeq \beta \cos \theta .
\end{gathered}
$$

According to (2) the incident particles produce the isotropic flux from the second entrance into the wormhole throat (in the rest frame of the second entrance) with the same energy $E^{\prime}$. In the case of an isotropic distribution of incident particles the mean change of the energy vanishes $\langle\cos \theta\rangle=0$. This means that for the isotropic background we have the same situation as in the case of $\mathrm{CMB}$, the motion of the absorbing end of the wormhole does not matter. The peculiar motion of the emitting throat entrance produces the effect analogous to KSZ effect, which is the common Doppler shift of the energy $\Delta E^{\prime} / E=\beta_{p}$, where $\beta_{p}=V_{p} / c$ is the projection of the wormhole velocity on the direction pointing out to the observer. For the spectrum (7) it can be described in terms of the respective shift of the value $\Delta E_{0} / E_{0}=\beta_{p}$ which admits both signs. The basic property of the spectrum (7) is that such a shift does not change the form of the spectrum. In the case $\tau_{w} \ll 1$ the form of the spectrum does not change also in next orders in $\beta_{p}$. For sufficiently dense clouds of wormholes $\tau_{w} \sim 1$ relativistic corrections include also terms of the form $\Delta E^{\prime} / E \sim m^{2} c^{4} / E^{2}$ which do produce distortions of the form of the spectrum but they are to small for high energies and can be neglected. The Doppler shift appears also in the case when the source of radiation moves and both effects merge. KSZ however somewhat smoothes the break in the spectral index at the energy $E \simeq E_{b} \simeq 0.9 \mathrm{TeV}$. Thus the basic effect of relic wormholes which admits observation is a small shift (positive or negative) of the value $E_{b}$ in high-energy cosmic rays.

In conclusion of the section we point out that such a mechanism (the generation of a shift of spectrum) works during the whole period of the evolution of the Universe. In 
particular, it works also at the time of photon decoupling and if there were such processes as dark-matter particle annihilation or decay, effects of scattering on wormholes should be imprinted in the spectrum.

\section{The scattering of CMB on a single torus-like wormhole}

In the case of a single wormhole we should account for the two important features. The first feature is the fact that a stable cosmological wormhole has the throat section in the form of a torus (Kirillov \& Savelova 2016). The simplest model of a torus-like wormhole is given by a couple of conjugated torus-like mirrors. Therefore, if such a wormhole is sufficiently big, then the simplest way to find it is to look for the direct imprints on CMB maps. Indeed, by means of KSZ effect a wormhole should produce a ring on CMB map that has a temperature which is slightly different from the background temperature. In particular, it was reported recently in (Meissner et.al. 2013), that there are, with confidence level 99.7 per cent, such ring-type structures in the observed cosmic microwave background. We hope that such structures could be imprints of cosmological wormholes indeed. In this case however more frequent structures should have elliptical form, since tori (wormhole throats) have random orientations in space.

The second important feature is that the scattering forward (i.e. absorption of CMB photons (1)) produces much bigger effect (since $k R \gg 1, k a \gg 1$, where $k$ is the wave-vector, $R$ is the largest, and $a$ is the smallest radiuses of the torus respectively). This effect corresponds to the standard diffraction on the torus-like obstacle. In the approximation $\mu=a / R \ll 1$, where $a$ is the smallest radius of the torus, we may use the flat screen approximation.

Let the orientation of the torus (the normal to the torus direction) be along the $\mathrm{Oz}$ 
axis, i.e. $m=(0,0,1)$. The cross-section depends on the two groups of angle variables, i.e. the two unit vectors $n_{0}\left(\phi_{0}, \theta_{0}\right)$ and $n(\phi, \theta)$. The vector $n_{0}=\left(\cos \phi_{0} \sin \theta_{0}, \sin \phi_{0} \sin \theta_{0}, \cos \theta_{0}\right)$ points to the direction of the incident photon (i.e., the wave vector is $k_{0}=\frac{\omega}{c} n_{0}$ ), while the vector $n$ corresponds to the scattered photons. Then the cross-section is given by

$$
\frac{d \sigma}{d \Omega}=\sigma_{R} \sin ^{2} \theta_{0} \frac{(k R)^{2}}{4 \pi}\left(\frac{1+\cos ^{2} \theta}{2}\right)|F|^{2},
$$

where $\sigma_{R}=\pi R^{2}$, and the function $F$ is

$$
F=(1+\mu)^{2} \frac{2 J_{1}((1+\mu) y)}{(1+\mu) y}-(1-\mu)^{2} \frac{2 J_{1}((1-\mu) y)}{(1-\mu) y}
$$

where $y=k R \xi$. We also denote

$$
\xi=\left(\sin ^{2} \theta+\sin ^{2} \theta_{0}-2 \sin \theta \sin \theta_{0} \cos \left(\phi-\phi_{0}\right)\right)^{1 / 2}
$$

and $J_{n}(y)$ are the Bessel functions. We also averaged $\sigma$ over polarizations. Let us expand the kernel $F$ by the small parameter $\mu \ll 1$ which gives

$$
F \approx 2 \mu\left(y\left(\frac{2 J_{1}(y)}{y}\right)^{\prime}+2 \frac{2 J_{1}(y)}{y}\right) .
$$

Using the property $\left(J_{\nu}(y) / y^{\nu}\right)^{\prime}=-J_{\nu+1}(y) / y^{\nu}$ and the identity $J_{2}(y)=\frac{2}{y} J_{1}(y)-J_{0}(y)$ we get $F \approx 4 \mu J_{0}(y)$ which gives

$$
\frac{d \sigma}{d \Omega}=8 \sigma_{R} \frac{(k a)^{2}}{4 \pi}\left(1-\cos ^{2} \theta_{0}\right)\left(1+\cos ^{2} \theta\right)\left|J_{0}(k R \xi)\right|^{2} .
$$

The intensity of the scattered radiation in the solid angle $d \Omega$ and in the interval of frequencies $d \nu$ is given by

$$
\frac{1}{I_{\nu}} \frac{d \Delta I_{\nu}}{d \Omega}=\frac{2 \sigma_{R}(k a)^{2}}{\pi}\left(1+\cos ^{2} \theta\right) \int\left|J_{0}(k R \xi)\right|^{2} \sin ^{2} \theta_{0} d \Omega_{0},
$$

where $I_{\nu}=c \rho(x)$ is the intensity of the incident black body radiation and $x=h \nu / k_{B} T_{C M B}$. The above expression improves the absorbtion part given by (11). Since $k R \gg 1$, it shows the presence of specific ring-type oscillations in the cross-section. Indeed, if we consider the normal fall of the incident photons, i.e., $\theta_{0}=0$, then we find $\frac{d \sigma}{d \Omega} \sim J_{0}(k R \theta)$. For sufficiently remote throats the value $R \theta$ is small and such oscillations should be imprinted in the diffraction picture of $\mathrm{CMB}$ in the form of rings. 


\section{Conclusion}

In conclusion we point out that in searching for KSZ signal from wormholes we meet two basic problems. First one is the need of independent observational effects related to wormholes which can be compared to KSZ. The simplest effect of such a kind we find, if we consider propagation of cosmic rays (of any origin) through the same region of space where we expect to observe KSZ. Wormholes were shown to produce an additional damping in cosmic rays (Kirillov et al. 2008) which is determined by the same optical depth (5) $\tau_{w}$. Thus, if there is a discrete source of a standard intensity, the optical depth can be directly measured. The damping is caused by the capture of some part of particles by wormholes. Particles captured are re-emitted (by the second entrance into wormhole throats) in an isotropic way which forms a diffuse halo around any discrete source. In the absence of peculiar motions of wormholes such a halo has the same energy spectrum. Peculiar motions cause a shift of the initial cosmic ray spectrum without a change of it's form. For example, random motions should somewhat smooth the detected break in the teraelectronvolt cosmic-ray spectrum of electrons and positrons (Ambrosi et al. 2017), while common peculiar motions simply produce an additional shift of the threshold value $E_{b}$. The Doppler shift of the spectrum can also be attributed to the motion of the source itself. Therefore, to disentangle KSZ and the motion of the source represents very difficult problem and such subtle effects require the further investigation.

Another possibility is to extract basic parameters (such as the density of wormholes $n_{w}$ and the characteristic cross- section $\sigma_{0}=\pi \overline{a^{2}}$ ) from the distribution of dark matter. For example, the behavior of dark matter in galaxies may fix two parameters e.g., see (Persic et.al. 1996; Kirillov \& Turaev 2006) by means of measuring the empirical Newton's potential. Indeed, in galaxies the distributions of dark and luminous matter strongly correlate (Donato et.al. 2004). This means the existence of a rigid relation 
$\rho_{D M}(k)=b(k) \rho_{v i s}(k)$, where $\rho(k)$ are Fourier transforms for dark and visible matter densities. Then from the observed distribution of dark matter in galaxies, e.g. see Gentile et.al. 2004; Weldrake et. al. 2003), we may retrieve the Newton's potential as (Kirillov \& Turaev 2006)

$$
\phi_{e m p}=-\frac{4 \pi G b(k)}{k^{2}},
$$

which describes the deviation from the Newton's law. Observations of rotation curves are fitted by the simple function $b(k)=\left(1+(R k)^{-\alpha}\right)$. At small scales $(R k \gg 1)$ it gives the standard Newton's law, while at large scales $R k \ll 1$ it transforms to the fractal law, or the logarithmic behavior. We point out that the correction observed in galaxies corresponds to the value $\alpha \approx 1$ and $R \sim 5 K p c$. These parameters can be related to the distribution of wormholes (Kirillov \& Savelova 2017) but this problem requires the further study.

The second problem is that in galaxies and clusters (as well in the hot X-ray gas) the KSZ effect on CMB based on wormholes mixes with that on other sorts of matter (dust, hot gas, etc.). The difference appears only in the second order in $V / c$ (6) which requires sufficiently high accuracy of observations. 


\section{REFERENCES}

Ambjorn J., Jurkiewicz J., Loll R., 2005, Phys. Rev. Lett., 95, 171301.

Ambrosi, G. et al. 2017, Nature, 552, 24475.

Battistelli E. S. et.al., 2016, Int. J. Mod. Phys. D, 25, 1630023.

Borriello A., Salucci P., Danese L., (2003) Mon. Not. R. Astron. Soc. 3411109.

Clement G., 1984, Int. Journ. Theor. Phys., 23, 335.

Conde-Saavedraa G., Iribarrema A., Ribeirob M.B., 2015, Physica A 417 332-344.

Donato F., Gentile G., Salucci P., 2004, MNRAS, 353, L17

Feng J.L., 2010, Annu. Rev. Astron. Astrophys. 48, 495-545

Gentile G., Salucci P., Klein U., Vergani D., Kalberla P., 2004, MNRAS, 351, 903

Grib A.A., Pavlov Yu.V., 2009, Gravitation and Cosmology, 15, 44-48.

Hand N., et.al., 2012, Phys. Rev. Lett. 109, 041101.

Hochberg, D.; Visser, M., 1998, Phys. Rev. Lett. 81, 746-749.

Kashlinsky A., Atrio-Barandela F., Ebeling H., 2011, ApJ, 732, 1.

Kirillov A.A., Turaev D., 2006, MNRAS, 371 L31.

Kirillov A.A., Savelova E.P., Shamshutdinova G.D., 2009, JETP Lett., 90, 599.

Kirillov A.A. \& Savelova E.P., 2011, MNRAS, 412, 1710.

Kirillov A.A. \& Savelova E.P., 2012, Phys. Lett., B 710, 516.

Kirillov A.A. \& Savelova E.P., 2015, Physics of Atomic Nuclei, 78, 1069-1073. 
Kirillov A.A. \& Savelova E.P., 2016, Int. J. Mod. Phys. D, 25, 1650075.

Kirillov A.A. \& Savelova E.P., 2017, Int. J. Mod. Phys. D, 261750145.

Kirillov A.A., Savelova E.P., Zolotarev P.S., 2008, Physics Letters B, 663 372-376.

Labini S. F., Montuori M., Pietronero L., 1998, Phys. Rep. 293, 66

Meissner K.A., Nurowski P., \& Ruszczycki B., 2013, Proc R Soc A, 46920130116.

Navarro J. F., Frenk C. S., White S. D. M., 1996, ApJ, 462, 563

Perez Bergliaffa S.E., Hibberd K.E., 2000, Phys. Rev. D62 044045.

Persic M., Salucci P., Stel F., 1996, MNRAS, 281, 27.

Planck Collaboration, 2016, A\&A, 594, A18

Planck Collaboration, 2016, A\&A, 594, A24

Sayers J., et. al., 2013, ApJ, 778, 52.

Savelova E.P., 2015, Gravitation and Cosmology, 21, 48-56.

Savelova E.P., 2016, Gen Relativ Gravit, 48:85.

Sunyaev R.A., Zeldovich Ya.B., 1980a, MNRAS, 190, 413.

SunyaevR.A., Zeldovich Ya.B., 1980b, Ann Rev A , 18, 537.

Weldrake D. T. F., de Blok W. J. G., Walter F., 2003, MNRAS, 340, 12 